\title{
Implementation of a semiautomatic method to design patient-specific instruments for corrective osteotomy of the radius
}

\author{
G. Caiti ${ }^{1}$ (I) J. G. G. Dobbe ${ }^{1}$ - A. C. Y. Loenen ${ }^{2,3}$ - M. Beerens ${ }^{4}$ - S. D. Strackee ${ }^{5}$. G. J. Strijkers ${ }^{1}$. G. J. Streekstra ${ }^{1}$
}

Received: 28 August 2018 / Accepted: 30 November 2018 / Published online: 10 December 2018

(c) The Author(s) 2018

\begin{abstract}
Purpose 3D-printed patient-specific instruments (PSIs), such as surgical guides and implants, show great promise for accurate navigation in surgical correction of post-traumatic deformities of the distal radius. However, existing costs of computer-aided design and manufacturing process prevent everyday surgical use. In this paper, we propose an innovative semiautomatic methodology to streamline the PSIs design.

Methods The new method was implemented as an extension of our existing 3D planning software. It facilitates the design of a regular and smooth implant and a companion guide starting from a user-selected surface on the affected bone. We evaluated the software by designing PSIs starting from preoperative virtual 3D plans of five patients previously treated at our institute for corrective osteotomy. We repeated the design for the same cases also with commercially available software, with and without dedicated customization. We measured design time and tracked user activity during the design process of implants, guides and subsequent modifications.

Results All the designed shapes were considered valid. Median design times $(\tilde{t})$ were reduced for implants $\left(\left(\tilde{t}_{I}\right)=2.2 \mathrm{~min}\right)$ and guides $\left(\left(\tilde{t}_{G}\right)=1.0 \mathrm{~min}\right)$ compared to the standard $\left(\left(\tilde{t}_{I}\right)=13 \mathrm{~min}\right.$ and $\left.\left(\tilde{t}_{G}\right)=8 \mathrm{~min}\right)$ and the partially customized $\left(\left(\tilde{t}_{I}\right)=\right.$ $6.5 \mathrm{~min}$ and $\left.\left(\tilde{t}_{G}\right)=6.0 \mathrm{~min}\right)$ commercially available alternatives. Mouse and keyboard activities were reduced (median count of strokes and clicks during implant design $\left(\left(\tilde{s}_{I}\right)=53\right.$, and guide design $\left(\left(\tilde{s}_{G}\right)=27\right)$ compared to using standard software $\left(\left(\tilde{s}_{I}\right)=559\right.$ and $\left.\left(\tilde{s}_{G}\right)=380\right)$ and customized commercial software $\left(\left(\tilde{s}_{I}\right)=217\right.$ and $\left.\left(\tilde{s}_{G}\right)=180\right)$.

Conclusion Our software solution efficiently streamlines the design of PSIs for distal radius malunion. It represents a first step in making 3D-printed PSIs technology more accessible.
\end{abstract}

Keywords Patient-specific instruments · Distal radius malunion · Computer-aided design · Semiautomatic design

G. Caiti

g.caiti@amc.uva.nl

1 Amsterdam UMC, Department of Biomedical Engineering and Physics, University of Amsterdam, Amsterdam Movement Sciences, 1105 AZ Amsterdam, The Netherlands

2 Laboratory for Experimental Orthopaedics, Department of Orthopaedic Surgery, CAPHRI Care and Public Health Research Institute, Maastricht University Medical Centre, Maastricht, The Netherlands

3 Orthopaedic Biomechanics, Department of Biomedical Engineering, Eindhoven University of Technology, Eindhoven, The Netherlands

4 Xilloc Medical B.V., Sittard-Geleen, The Netherlands

5 Amsterdam UMC, Department of Plastic, Reconstructive and Hand Surgery, University of Amsterdam, Amsterdam Movement Sciences, 1105 AZ Amsterdam, The Netherlands

\section{Introduction}

Malunion is a complication that arises when fractured bone segments unite in a suboptimal position [1]. As a result, the malunited bone is characterized by non-anatomical bending, twist and/or shortening. In the case of radius malunion, this deformity can compromise the natural biomechanics of the distal radioulnar and radiocarpal joints and lead to a loss of wrist function and forearm rotation [2]. When the malunion is symptomatic, it is treated by corrective osteotomy surgery in which the bone is cut at the level of the deformity and subsequently realigned and fixated in a more anatomical position [3]. Since the outcome of the surgery is directly related to bone realignment $[4,5]$, accurate surgical planning is fundamental.

In the last decades, three-dimensional (3D) computerassisted techniques in preoperative planning of corrective 
osteotomy of the radius have been established [6-8]. In these $3 \mathrm{D}$ techniques, relying on a bilateral computer tomography (CT) scan of the radius, the optimal correction parameters are calculated in six degrees of freedom by using the mirrored contralateral bone as reference [9-11]. The correction parameters can then be used to virtually perform the osteotomy and to reposition the bone segments.

Despite the growing popularity of 3D planning techniques, one of the major challenges is the transfer of the preoperative plan to the patient during surgery. A promising solution is the use of patient-specific instruments (PSIs) such as surgical cutting guides and implants designed to match the patient's anatomy. The cutting guide is used to physically transfer the osteotomy planning, while the plate, which fits the deformed bone segments in the planned alignment, is subsequently used to fixate the bone segments $[10,12]$. These custom guides and implants (PSIs) can be modeled using computeraided design (CAD) software during preoperative surgical planning and fabricated via a computer-aided manufacturing (CAM) technology, such as 3D printing [3, 9, 10, 13-17].

Yet, despite the auspicious clinical results [12], PSIs are still not the first choice for every surgery mainly because of the costs and time related to PSIs design and manufacturing. Due to the complexity and scarce automation of commercially available standard CAD software [18, 19], design of PSIs can be an exhausting process taking up to several hours [20]. Although initiatives have been proposed to customize standard CAD software to automatize repetitive tasks (e.g., via Visual Programming), more automated techniques targeting specific PSI design tasks may be beneficial to make the design process more efficient.

Therefore, the purpose of the present study is to introduce and evaluate a novel method that reduces the complexity and speeds up the design of PSIs for corrective osteotomy of the radius. Hence, we compared the time and the user interaction required when designing PSI using a standard, partially customized and the proposed dedicated CAD software solutions.

\section{Methods}

Design of PSIs for corrective osteotomy requires preoperative position planning based on the contralateral healthy bone. In this study, preoperative planning was performed in 3D with existing custom-made software, as described by Dobbe et al. [9, 21, 22]. In brief, we obtain a 3D polygon model of the affected radius by image segmentation (level set segmentation initialized by region growing) from a CT scan of the patient's forearm. Distal and proximal segments are subsequently clipped, excluding the deformed region. Reg- istration of these clipped segments to the mirrored CT image of the contralateral healthy bone (Fig. 1a) results in a correction matrix $M_{\mathrm{c}}$ which brings the distal bone segment to the planned position. The surgeon then chooses an osteotomy plane (Fig. 1b). The distal segment resulting from the virtual osteotomy is repositioned using $M_{\mathrm{c}}$ (Fig. 1c) [1, 2].

In this study, results of the preoperative plan were considered as starting point for PSI design. These consisted of a 3D virtual model in the stereolithographic format (STL) of the affected bone, the osteotomized bone segments in the affected position, an additional version of the distal bone segment in the planned position and the correction matrix $M_{\mathrm{C}}$. The next paragraphs describe the three selected CAD software solutions (standard, partially customized and dedicated) to create guides and customized plates and the evaluation method that was used to compare them.

\section{PSI design using standard CAD software}

As a standard CAD software for PSI design, we evaluated 3-matic (Materialise, Leuven, Belgium), an established PSI design software package already adopted in many clinical studies [23, 24]. In this software, several manual operations are required to accomplish the task of designing PSIs for corrective osteotomy. The adopted workflow is summarized in Fig. 2a. We started the design of the implant by importing the STL datasets of the proximal and the distal bone polygons in the planned relative position. The sweep-loft function was then used to interpolate in-between these virtual bone segments. "Push and pull" manual operations were then required to locally smooth the interpolated region. On the surface of this corrected bone model, we manually positioned and oriented lines representing drill trajectories. A 2D sketch of the implant footprint was manually drawn and projected onto the corrected bone surface to select the implant footprint including the screw locations. The footprint was extruded, and the edges were smoothed. Finally, drill holes were created by subtracting cylinders of a specified diameter at locations indicated by the drill lines.

In order to create the surgical guide, the repositioned distal segment had to be registered to the original position (via point-cloud fitting). This enabled retrieving the transformation parameters (which, in this case, could not be provided via the transformation matrix) to bring the distal drilling lines to the affected state of the bone. After importing the STL of the affected bone, a 2D sketch, including the drilling line insertion points, was drawn onto its surface to select the guide footprint. The same steps mentioned before were then executed onto the guide footprint. Finally, a slit of thickness $0.8 \mathrm{~mm}$ was created centered around the previously chosen cutting plane. 
Fig. 1 a During position planning a distal and proximal segments of the affected bone are registered to the mirrored image of the healthy bone, here shown in a multi-planar reconstruction (MPR) view; b the surgeon chooses the osteotomy plane; $\mathbf{c}$ the distal segment is repositioned from the affected (red) to the planned (green) position using $M_{\mathrm{c}}$
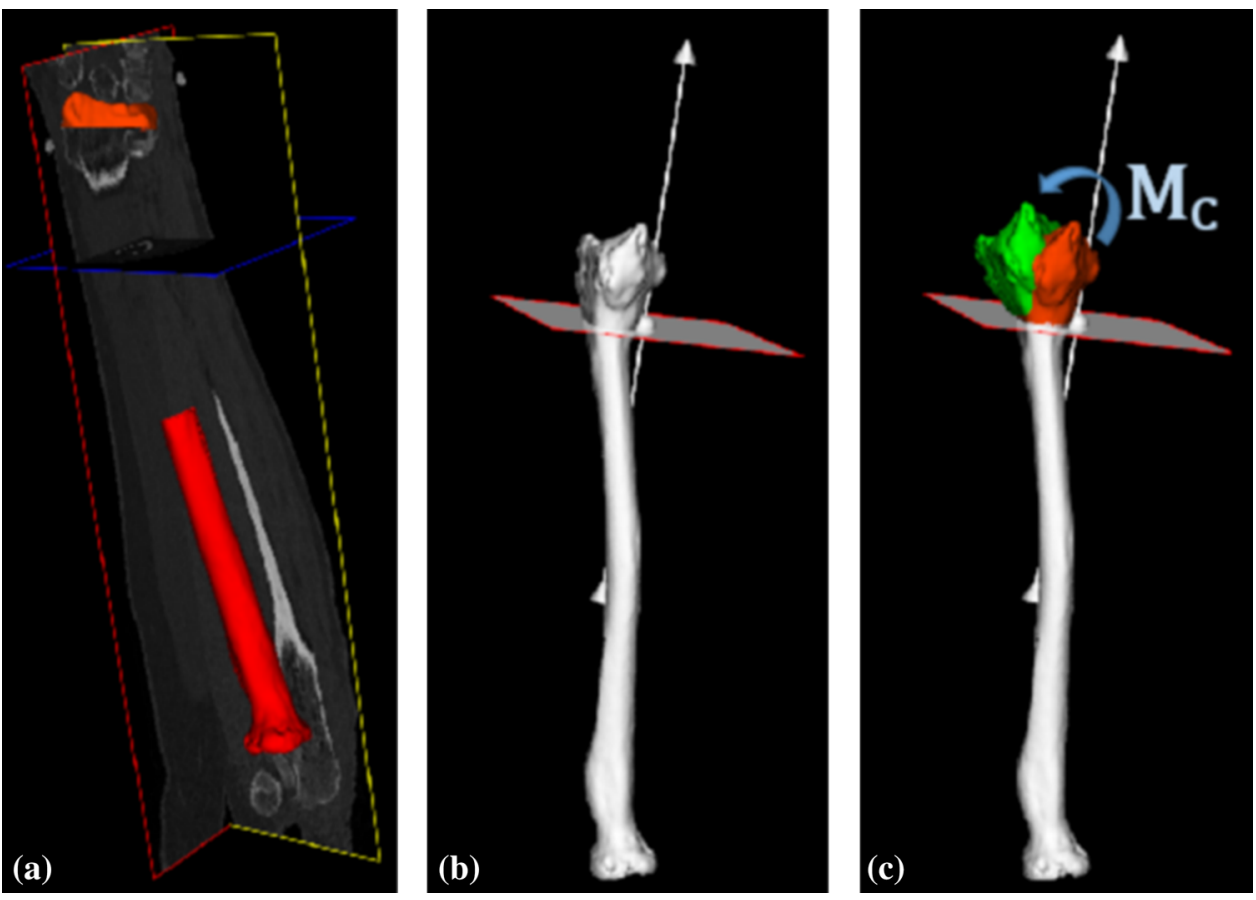
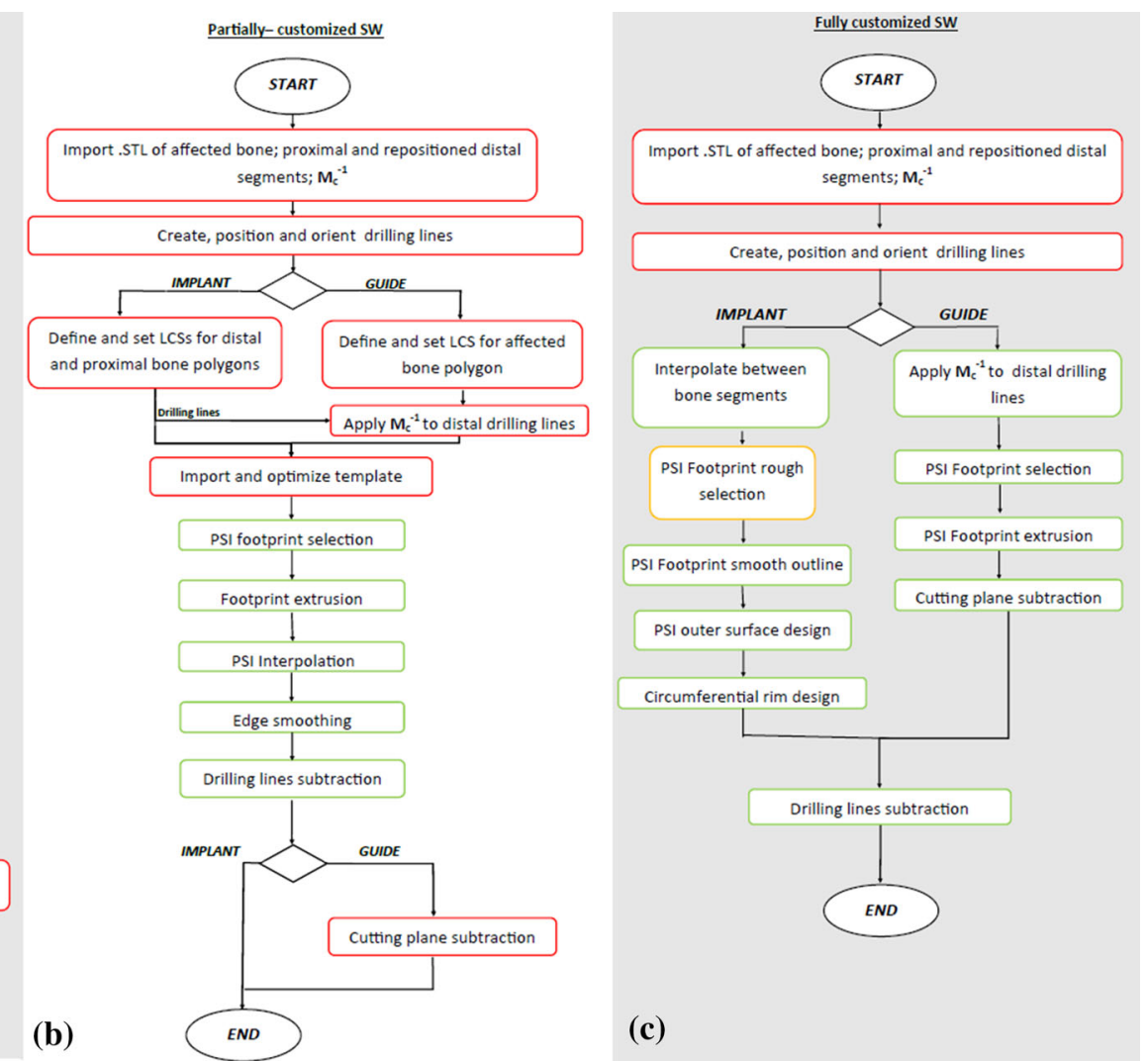

Fig. 2 Workflow for each of the three investigated CAD solutions. Red blocks represent manual operations and green blocks represent automatic operations. a Materialise 3-matic-adopted workflow; b Siemens NX partially customized solution; $\mathbf{c}$ the proposed semiautomatic solu- tion, the only manual step is the definition of the drilling lines. During the rough footprint selection, adjusting the size of the projection box is performed semiautomatically (orange block) 
Fig. 3 a Overview of the predefined 2D sketch for the distal segment. A local coordinate system (LCS) is used to position the sketch. The sketch can be optimized by dragging the vertices (an analogue sketch is used for the proximal bone segment; $\mathbf{b} 2 \mathrm{D}$ distal and proximal sketches are projected onto the respective target bone polygons, extruded into 3D plate segments and merged by interpolation

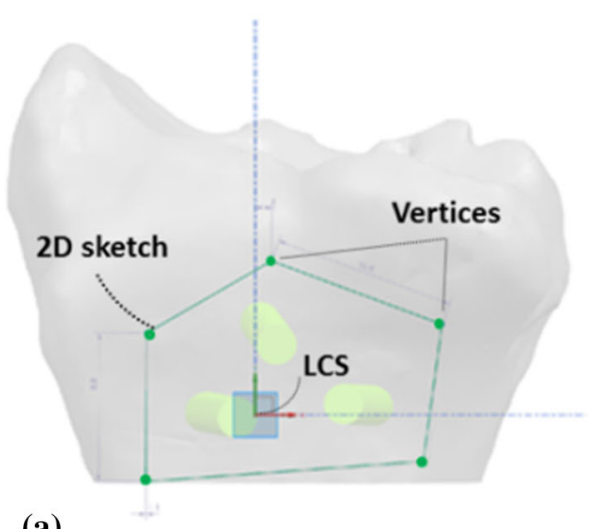

(a)

(b)

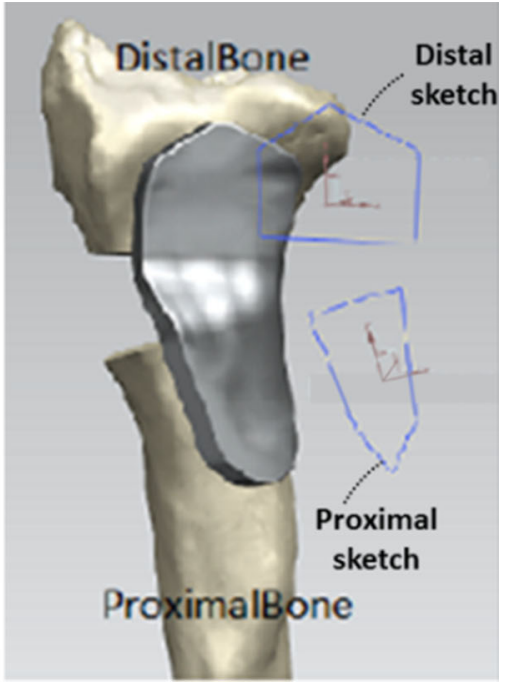

\section{PSI design using customizable CAD software}

A partially customized CAD software solution was developed with Siemens NX (formerly known as NX Unigraphics; Siemens PLM software, Plano, TX, USA). Siemens NX is a multi-purpose industrial design, simulation and manufacturing software providing a collection of Parametric Product Models under the name "Product Template Studio." Every Parametric Product Model corresponds to a function that can be manually activated in NX. In "Product Template Studio," the user can create, by Visual Programming, custom interfaces by linking different Parametric Product Models to automatize the design. In this environment, we created two separate applications, respectively, for the implant and guide design. The overall workflow of implant and guide design applications are summarized in Fig. $2 \mathrm{~b}$ and detailed below.

Preliminary step Before running the design applications, definition of the drill trajectories is required. These are created by manually positioning virtual lines.

Implant design application A local coordinate system (LCS) has to be defined for both the distal and proximal bone parts. These LCSs will be used to position and project two predefined 2D implant sketches adequately onto the repositioned bone model (Fig. 3a). The 2D sketches can then be manually adjusted by dragging predefined vertices.

After the sketches have been shaped as desired, the automatic design procedure is started. In brief, both proximal and distal sketches are projected onto the target bone to retrieve two partial implant footprints. The partial footprints are then extruded and merged by interpolation to create the plate (Fig. 3b). Corners around the plate are blended to avoid sharp edges. To finish implant design, screw holes were created centered around the predefined drilling lines.
Guide design application The application for surgical guide design uses almost the same consecutive commands as described for the implant design. However, the interpolation step is not required for the surgical guide design. The local coordinate system (LCS) is defined onto the surface of the affected bone polygon. Again, a predefined 2D sketch for the guide is positioned at the origin of the LCSs. After manual optimization of the 2D sketch, the automatic design procedure is started with the same steps as previously mentioned. The cutting slit is manually created by using the cutting plane position. To realize the correct positions for the drilling holes on the surgical guide, the inverse of the correction matrix $\left(M_{\mathrm{c}}^{-1}\right)$ is used to transform (a copy of) the distal lines representing the distal drilling lines, on the correct positions with respect to the surgical guide. The slit of thickness $0.8 \mathrm{~mm}$ was created centered around the predefined cutting plane.

\section{PSI design with dedicated software}

A semiautomatic CAD wizard was added to the existing custom software for surgical planning [21, 22]. The wizard was implemented in the $\mathrm{C}++$ programming language (Visual Studio 2013, Microsoft, Redmond, WA). The Visualization Toolkit (VTK 7.0.0) was used for modeling and visualization in 3D space. Sequential steps in the design approaches are summarized in the workflow of Fig. 2c.

Drill-line placement A virtual model of the osteotomized bone segments in the planned position is created by application of the correction matrix $M_{\mathrm{c}}$ (Fig. 4a). The user selects a number $n$ of screw insertion points by tagging them on this model. Screw trajectories represented by lines are automatically proposed in the direction of the local surface normal of the bone model although the line can be adjusted manually (Fig. 4b). 


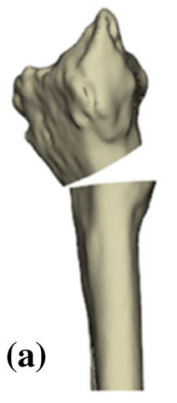

(b)

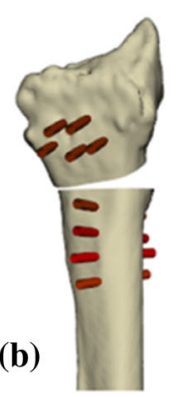

(c)

Fig. 4 Proposed workflow in automated design of custom implants: a reduced bone model with the segments in the planned position; $\mathbf{b}$ on this model, the user selects the screw insertion points on, e.g., the volar side of the radius. The lines represent screw trajectories; $\mathbf{c}$ the average
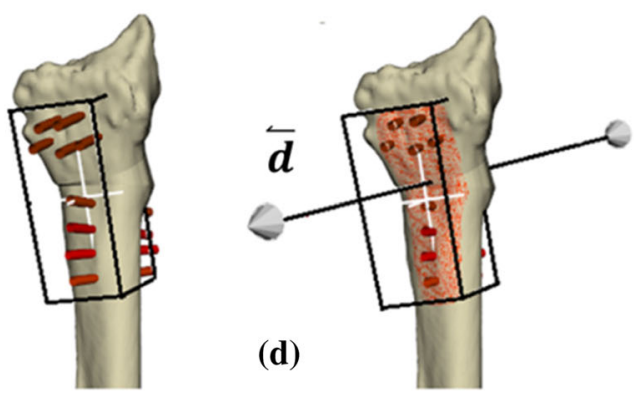

cylinder directions and the screw insertion points are used to automatically size and orient a virtual box to select the implant footprint on the corrected bone model; $\mathbf{d}$ a regular grid of point from one side of the virtual box is projected onto the surface of the corrected bone model

The position of the intermediate point $p_{i}^{\prime}$ determines the orientation of the plane in which the $b$-spline resides and the bulge of the circumferential rim. Initially, the position of $p_{i}^{\prime}$ is

$p_{i}^{\prime}=p_{i}+\left(b \cdot \vec{e}+\frac{t}{2} \cdot \vec{d}\right)$

where $b$ and $t$ are user-defined scalars representing the bulge of the implant and the implant thickness; $\vec{e}$ is a bisecting unity vector starting at $p_{i}$ splitting the external counterclockwise angle $(\alpha)$ between the vectors $\overrightarrow{p_{i} p_{(i-1)}}$ and $\overrightarrow{p_{i} p_{(i+1)}}$ in two (Fig. 5c).

However, design of circumferential rims in the procedure above may not work in case the outline is locally strongly concave, i.e., $\alpha_{i}<180^{\circ}$ in which case intersections of $b$ splines can occur depending on the edge bulge $b$ (see Fig. 5c).

Therefore, we implemented an iterative algorithm that, for a user-defined value of $b$, checks for $b$-spline intersections and reduces concavity by smoothing the footprint outline, until the $b$-splines no longer intersect. Finding intersections between each couple of segments was implemented as described in [25]. Whenever an intersection is found, we applied a simple central moving average filter to the outline points, which has the effect of repositioning each point based on the position of its two boundary neighbors. Based on the new position of the points $p_{i}$, points $p_{i}^{\prime}$ are recalculated and intersections are checked. This procedure is iterated until no more intersections are found. Since the $b$-splines that define the circumferential rim do not depend on where along the projection axis $(\vec{d})$ each point $p_{i}$ is found, smoothing of the plate outline is implemented in 2D. The 2D smoothed contour is then reprojected onto the bone model, and the surface of the bone contained inside the 3D contour is selected as the new implant footprint.

Outer surface smoothing A copy of the inner surface is smoothed and translated over a distance equal to the implant 


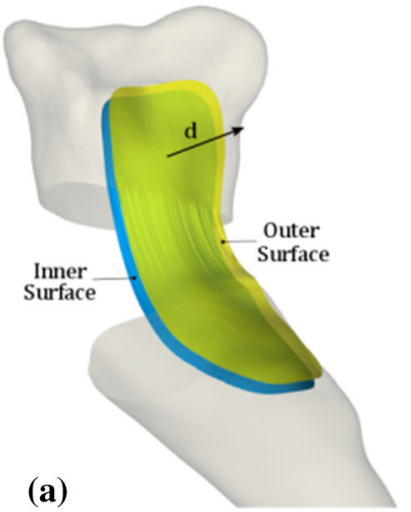

Fig. 5 Principle of the creation of an implant with a smooth circumferential rim. a The outer surface is a translated and smoothed version of the inner surface; $\mathbf{b}$ the bulging shape of the circumferential rim is obtained by fitting a cubic $b$-splines through each boundary point $p_{i}$, its corresponding copy on the outer surface $\left(p_{i}^{\prime \prime}\right)$ and an intermediate point

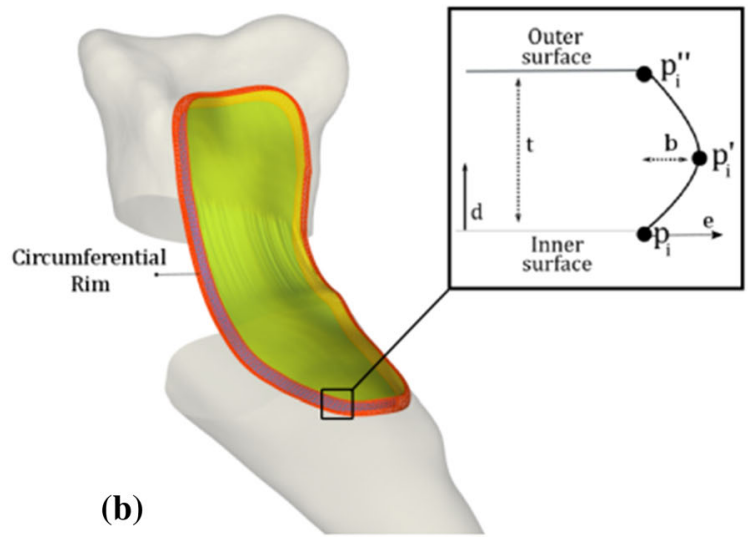

(c)

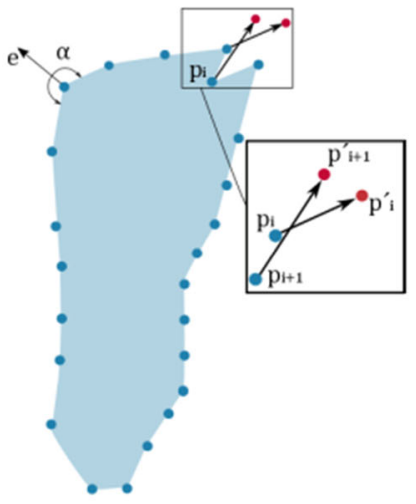

thickness $t$ (default $2.4 \mathrm{~mm}$ ) in the direction $(\vec{d})$ and represents the top surface of the plate. Smoothing of the top surface is accomplished by Laplacian smoothing (15 iterations) [26, 27]; the new position of each interior mesh points $q_{i}$ after smoothing is calculated as

$q_{i}=q_{i}+\lambda \cdot \Delta\left(q_{i}\right)$

$\Delta\left(q_{i}\right)=\frac{1}{\left|N_{i}\right|} \cdot\left(\sum_{j \in N_{i}} q_{j}\right)-q_{i}$

with $N_{i}$ being the set of the connected neighboring points to $q_{i}$ and $\lambda=0.3$ being the relaxation factor.

Subtraction of drilling lines The final step in the implant design consists in creating the screw holes in the plate by Boolean subtraction of the drilling lines from the implant.

Surgical guide modeling Once the design of the implant is completed, the guide design is subsequently started. The first step in the guide modeling is transforming the drilling holes (that are already defined in the plate design method) back to the affected bone model through the inverse of the correction matrix $M_{\mathrm{c}}^{-1}$. We use the same methodology described before for automatically sizing a virtual box and selecting the bone surface for fitting the guide based on the screw insertion points, to select the surface of the affected bone where the cutting guide will be positioned. This time, the selecting box is slightly oversized to better enclose the bone, which ensures a better fit and easier guide positioning during surgery [15]. The selected guide footprint is directly extruded, and a cutting slit and screw holes are included into the guide by Boolean subtraction. $\left(p_{i}^{\prime}\right)$. The distance $b$ determines the bulge of rim of the implant; $\mathbf{c}$ simplified sketch representing the $2 \mathrm{D}$ projection of the points making up the boundary of the implant footprint. Vector $\vec{e}$ is a bisecting unity vector, which splits the angle $\alpha$ between two consecutive boundary points. Intersections of $b$-splines can occur in case $\alpha<180^{\circ}$

\section{Laboratory study}

A laboratory experiment was performed to evaluate the feasibility to print the PSI designed with the novel method and to use it for bone repositioning. 3D models of an affected bone, the corresponding PSIs and the corrected model (reference model) were 3D printed in Polycarbonate-ISO (PC-ISO) (Fig. 6). Printing was performed with a Fortus $450 \mathrm{mc}$ fused deposition modeling printer (Stratasys, Eden, Minnesota, USA). Accuracy of the printer was $\pm 0.127 \mathrm{~mm}$ in all the printing directions. A mock surgery was performed via the guide and the implant, as previously described in [9], onto the plastic model. CT images of the corrected bone and of the reference model were then acquired. The achieved alignment was finally compared to the reference model as described in the previous section by segmentation and registration of the distal and proximal bone segments. Residual positioning errors were expressed in 6 DoF.

\section{Validation experiments}

Three biomedical engineers were recruited, each with experience in implant design with one of the three considered design methods. Preoperative virtual 3D plans of five patients were selected that were previously treated at our institute for corrective osteotomy using a patient-specific implant. Preoperative plans were provided to the participating engineers (STL models of bone segments, the cutting plane and the correction matrix $M_{\mathrm{C}}$ ). Approximate screw positions and drilling line orientations were provided to the participants, via $2 \mathrm{D}$ sketches. This simulated the actual qualitative transfer of information between surgeons and engineers in the design stage. For every patient case, the engineers were asked to 


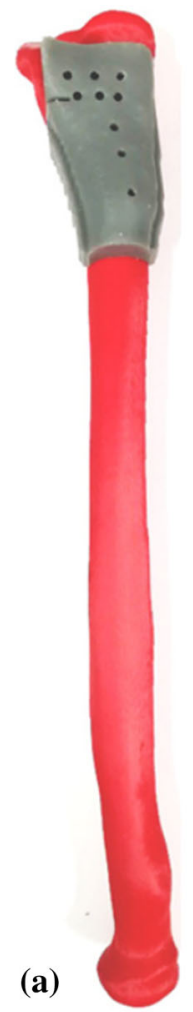

(b)

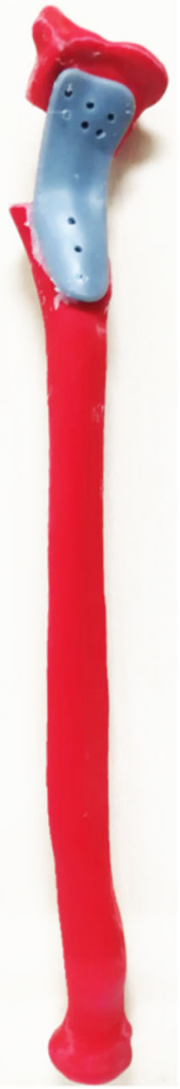

(c)

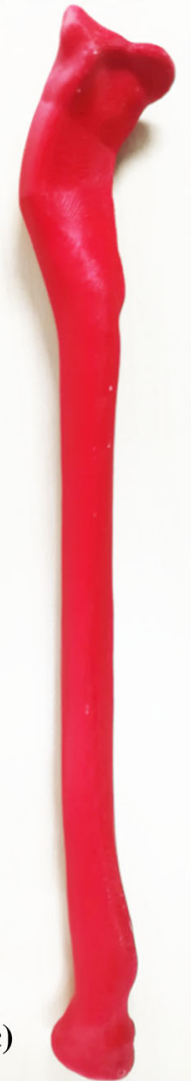

Fig. 6 3D-printed models used in the laboratory study: a affected bone models with cutting and drilling guide; $\mathbf{b}$ after cutting and drilling screw holes in the affected bone model, the patient-specific plate was glued to the resulting bone segments; $\mathbf{c}$ corrected bone model used as a reference in the laboratory study to calculate residual repositioning errors

design a patient-specific volar radius plate and its companion surgical guide featuring the cutting slit and the drill holes required for plate positioning. Since PSI design is often an iterative process, due to modifications requested by the surgeon and/or recommended after strength calculations using finite element analysis, after completing the design task, the engineers were asked to modify the screw configuration of the osteosynthesis material.

Measurements In each procedure, design time, the number of mouse movements and the number of keyboard strokes were recorded from the beginning until the end of the design process through Recorder User Input (RUI) [28], a publicly available logging tool. Tracked mouse movements were also converted into a single image using IOGraph (IOGraph V1.0.1, (C Anatoly Zenkov \& Andrey Shipilov, 2010-2018), which enabled illustration of the complexity of the design task in a graphical fashion. To check if the activity level of the different users during the tasks was comparable, we also calculated the mouse velocity and the number of clicks and strokes per minute of activity. The validity of all the designed

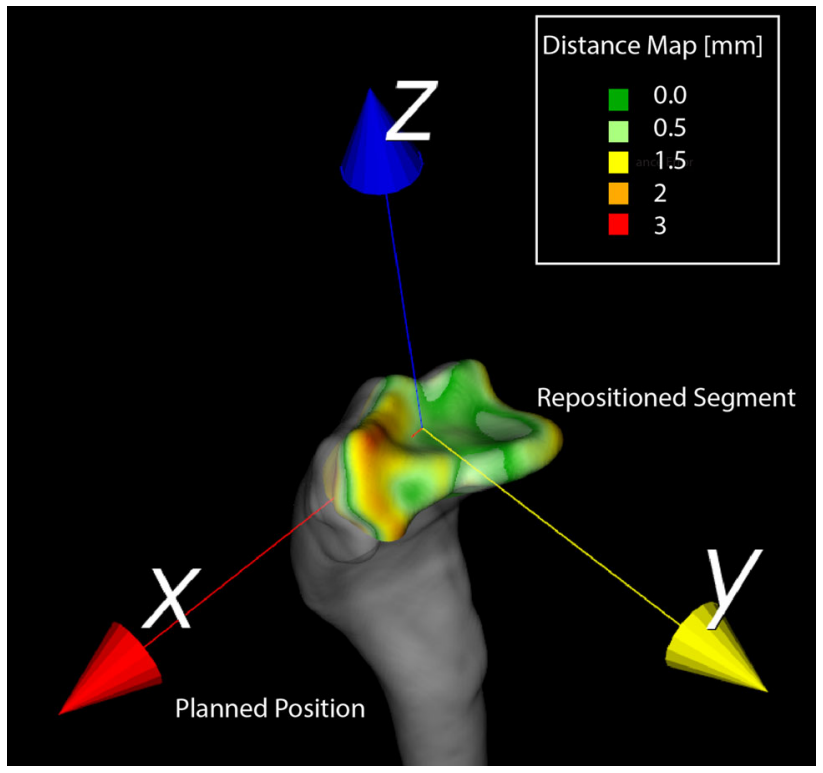

Fig. 7 3D reconstruction of the corrected bone and of the repositioned distal segment. Colors on the distal segment represent the nearest neighbor distance between points in the planned and achieved positions

PSI shapes was preliminary assessed by an experienced hand surgeon (SDS).

\section{Results}

Correction using the PSIs designed with the novel method was achieved in the mock surgery with residual 6 DOF errors expressed in terms of an anatomical coordinate system (Fig. 7) of: $\left(\Delta_{x}, \Delta_{y}, \Delta_{z}\right)=(-1.68,1.59,-0.15) \mathrm{mm}$ and a rotation error of $\left(\Delta_{\varphi_{x}}, \Delta_{\varphi_{y}}, \Delta_{\varphi_{z}}\right)=(-0.57,2.92,3.60)^{\circ}$. Figure 7 shows a color map representing the positioning error calculated as the nearest distance between the planned and achieved positions for every point in the distal segment.

After qualitative examination, all the designed PSI shapes were considered plausible and in agreement with the provided 2D preoperative planning. An example of the designed PSIs with the each of the design methods is shown in Fig. 8.

Results from user activity tracking in all the five design cases with the different software are shown in Fig. 9. For all the three software solutions, most of the time (42\%) was spent on the initial implant design. We evaluated the overall design of an implant, guide and subsequent modification and considered the average parameters as recorded for five cases using the standard software as reference. With the customized commercial software, the average design time, travelled mouse distance and keyboard and mouse strokes were reduced by $46 \%$; $54 \% ; 67 \%$ and by $84 \%$; $86 \%$; $90 \%$ with the proposed dedicated method. No training effect was visible. 

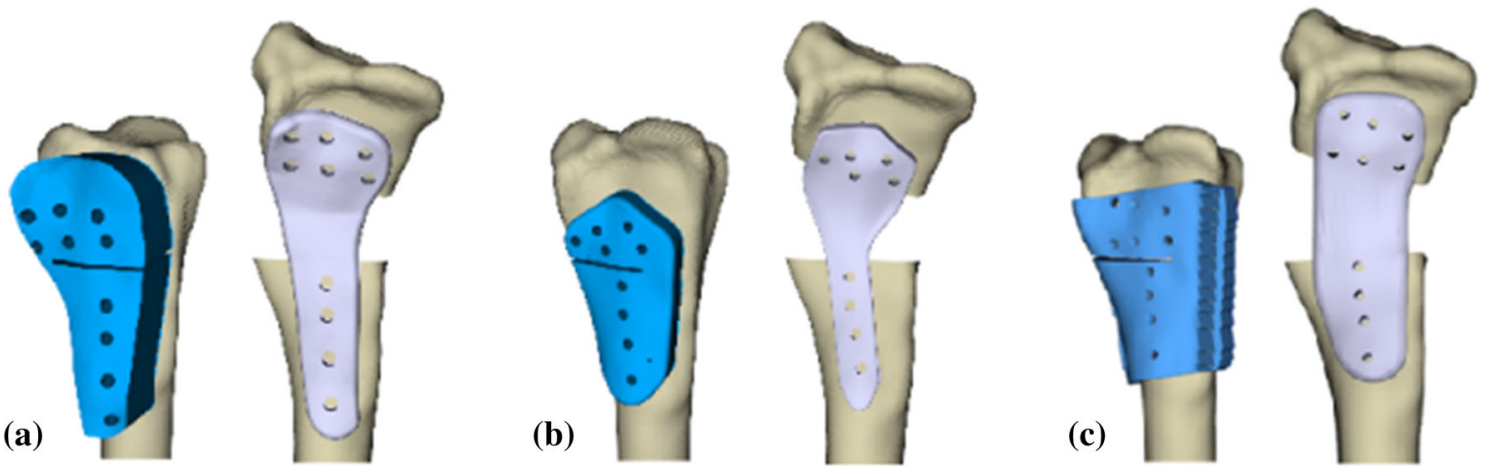

Fig. 8 Resulting implant and companion guide for the same patient case (C4) with the standard (a), the partially customized (b) and dedicated (c) software solutions

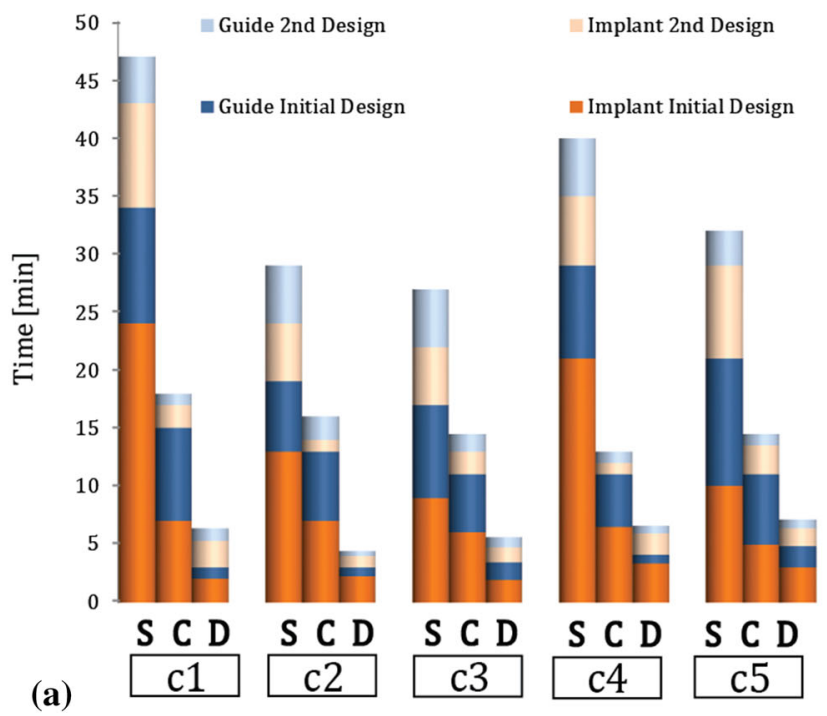

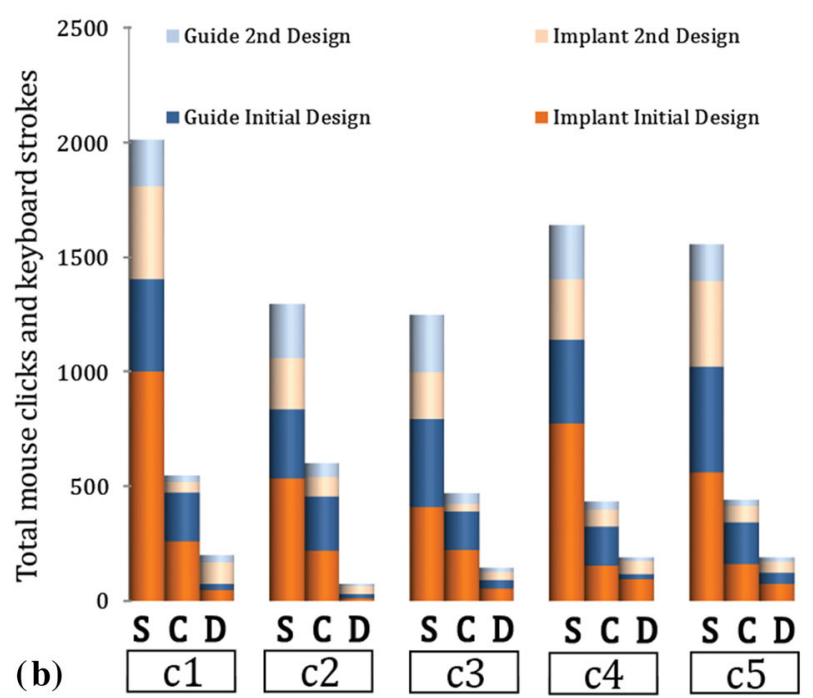

Fig. 9 We designed PSIs for five patient cases with the standard (S), partially customized (C) and Dedicated (D) CAD software solutions. For each of the five design cases (C1-C5), we recorded a the design time; $\mathbf{b}$ the number of mouse clicks and keyboard strokes and $\mathrm{c}$ we

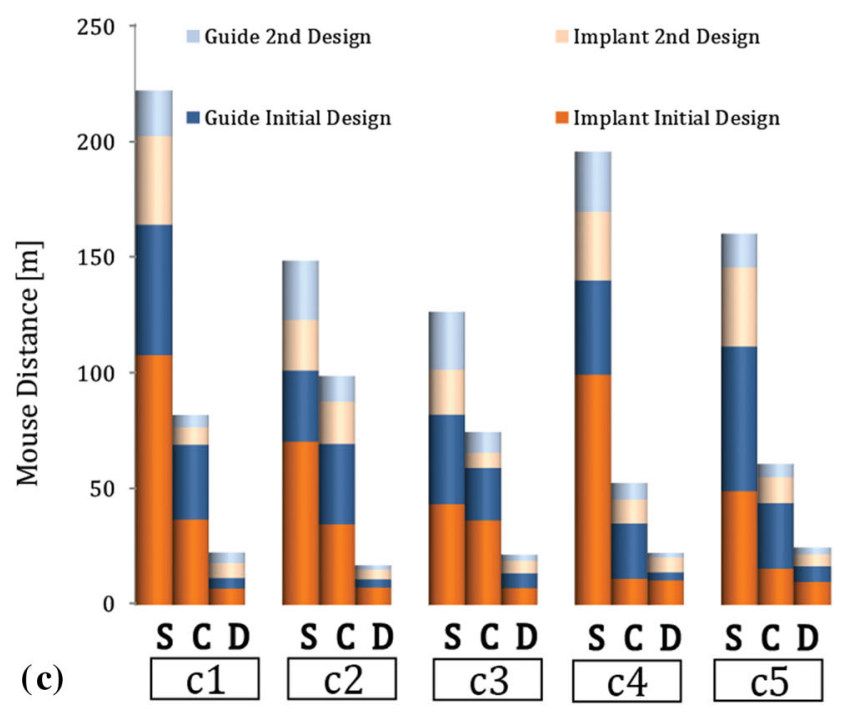

calculated the distance traveled by the mouse. These parameters were recorded during the initial design of the implant and guide, and during modification of the PSIs 

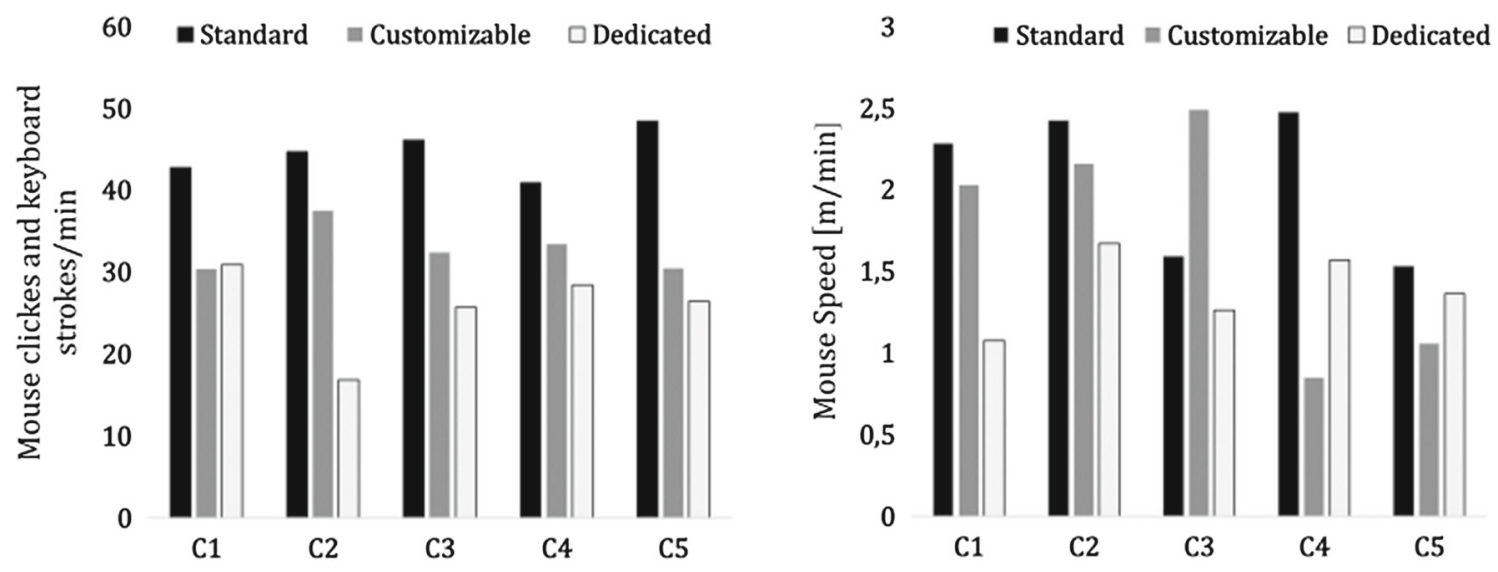

Fig. 10 Operator activity in terms of mouse clicks and keyboard strokes and mouse speed $(\mathrm{m} / \mathrm{min})$ during the five cases using the different design solutions
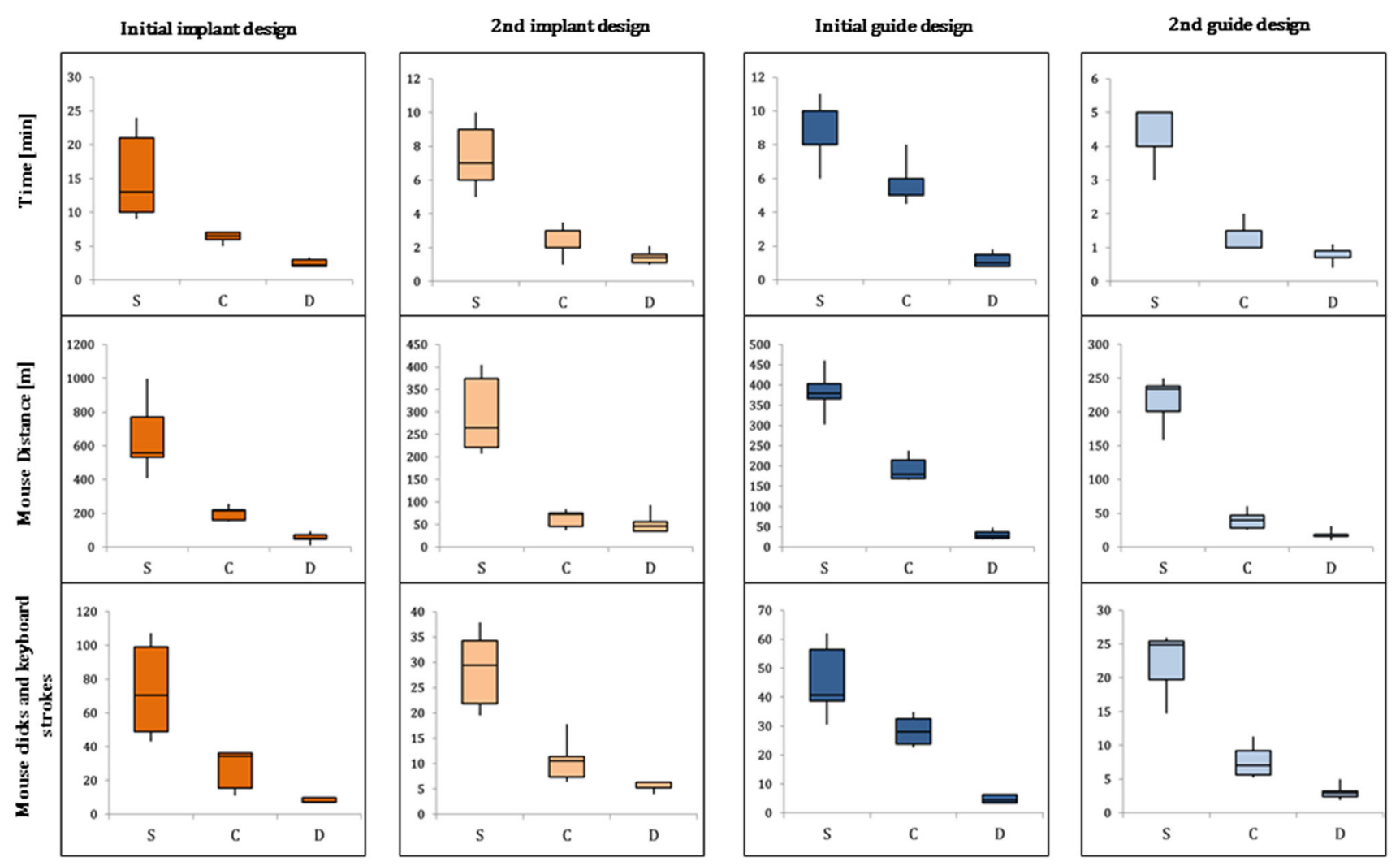

Fig. 11 Box plots represent the median and IQR of the measured variables (design time, number of mouse clicks and key strokes) across the five patient cases with the standard (S), partially customized (C) and dedicated (D) CAD software solutions

We found similar levels of user activity as shown in Fig. 10 during the design tasks.

Summary statistics of the recorded parameters (median and interquartile ranges (IQR)) calculated after pooling the five designed activity for each software is visualized in Fig. 11. Decreased IQR of time, clicks/stokes count and mouse distance show a reduced variability of design time and user activity across the cases in the custom and the dedicated solutions.

Tracked mouse movements' images of the design activity of the participants (Fig. 12) also visualize a more reproducible pattern of interaction with the proposed dedicated solution. 
Fig. 12 Picture resulting from mouse movements in the screen during the five designs tasks with a the standard, $\mathbf{b}$ the partially customized and $\mathbf{c}$ the dedicated CAD software solutions. The figure is subdivided into three panels, each representing a computer monitor. The central screen was dedicated to PSI design. The other monitors were used for the activity tracking applications

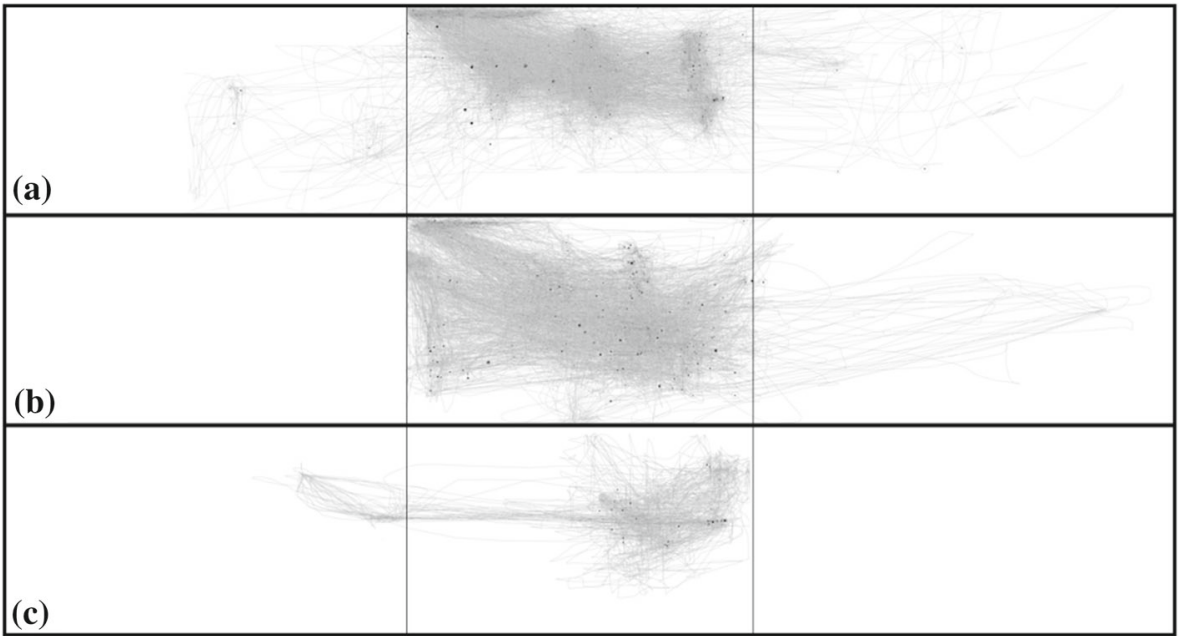

\section{Discussion}

Thanks to the recent technological advances, use of 3Dprinted patient-specific instruments including metal implants is gaining popularity and feasibility in the orthopedic field $[19,29,30]$. However, one of the shortcomings often reported in the medical literature is the time to process PSIs [18]. In general, the PSIs design relies on the collaboration between a surgeon and an engineering company or department [31]. Since computer-aided PSI design is usually a time-consuming process, several meetings between the surgeon and the design engineer are needed before the implant is satisfactory.

In the current study, we made a first step to automate PSI design for the distal radius. The residual positioning error as observed in the laboratory experiment was in agreement with the error as reported by Dobbe et al. in a larger laboratory study [9]. With our proposed design method, the median time to design an implant and a guide was $4.5 \mathrm{~min}$ which included a modification cycle. This was significantly reduced compared to using standard commercially available software. The shorter design time of the proposed methodology may enable designing PSIs in a single online session between surgeon and engineer. This could render PSI design less expensive and more accessible. Our method also showed a reduced variability in design time and in user interaction across the patient cases.

To our knowledge, this is one of the few studies that recorded the burden of design PSIs. Chen et al. already [32] proposed a general software package for the semiautomatic design of surgical templates such as surgical guides for corrective osteotomy. They reported $12 \mathrm{~min}$ to design an initial guide. With our proposed design method, the median time to design an initial guide was $1 \mathrm{~min}$.

In this study, we focused on the efficacy of the design methodologies and not on additional steps in the workflow, such as strength analysis or production of the PSIs, which can be considered a limitation of our study. Furthermore, in this paper, we did not consider the time related to production. However, with the rapid technological improvements, a dramatic decrease in the manufacturing time is expected. We nevertheless recommend investigating ways to improve these additional steps in the entire workflow of creating patientspecific PSIs.

Although the proposed method worked satisfactorily for the presented cases, we acknowledge that cases exist where the proposed method may perform poorly, such as in the presence of a hole or a large intrusion in the corrected model. Projection (see "PSI design with dedicated software" section) on such a surface would generate a non-feasible implant footprint. This can be overcome, however, by manually adjusting the depth of the virtual box that is used to define the footprint (see "PSI design with dedicated software" section) and by limiting the projection of points to the opposite face of the box instead of to the bone surface, thus generating an implant smoothly bridging possible intrusions.

During the design experiments, the participants were aware of being tracked. Therefore, the design times could be an underestimation of the design time that is valid in normal daily practice. Similar design experiments were also used by Egger et al. [33] to demonstrate the efficacy of a software package for cranial defect restoration. However, in our case it was considered not useful to compare the shape differences of implants designed with different methodologies since different implant shapes can be used for the same purpose of bone repositioning in corrective osteotomy surgery.

\section{Conclusion}

We presented a novel semiautomatic method for the design of patient-specific implants and associated cutting guides to be 
used in corrective osteotomy of the radius. Compared to the existing standard and partially customized CAD software, the novel method reduces times and interaction required in the design.

Acknowledgements This work was supported by Eurostars-2, ProjectID 9809, PERSONAL.

\section{Compliance with ethical standards}

Conflict of interest The authors declare that they have no conflict of interest.

Ethical approval All procedures performed in studies involving human participants were in accordance with the ethical standards of the institutional and/or national research committee and with the 1964 Declaration of Helsinki and its later amendments or comparable ethical standards.

Retrospective studies Formal consent is not required for this type of study.

Informed consent Informed consent was obtained from all individual participants included in the study.

Human and animal rights This article does not contain any studies involving animals performed by any of the authors.

Open Access This article is distributed under the terms of the Creative Commons Attribution 4.0 International License (http://creativecomm ons.org/licenses/by/4.0/), which permits unrestricted use, distribution, and reproduction in any medium, provided you give appropriate credit to the original author(s) and the source, provide a link to the Creative Commons license, and indicate if changes were made.

\section{References}

1. Peterson B, Gajendran V, Szabo RM (2008) Corrective osteotomy for deformity of the distal radius using a volar locking plate. Hand (N Y) 3:61-68. https://doi.org/10.1007/s11552-007-9066-y

2. Bushnell BD, Bynum DK (2007) Malunion of the distal radius. J Am Acad Orthop Surg 15:27-40

3. Byrne A-M, Impelmans B, Bertrand V, Van Haver A, Verstreken F (2017) Corrective osteotomy for malunited diaphyseal forearm fractures using preoperative 3-dimensional planning and patient-specific surgical guides and implants. J Hand Surg Am 42:836.e1-836.e12. https://doi.org/10.1016/j.jhsa.2017.06.003

4. Croitoru H, Ellis RE, Prihar R, Small CF, Pichora DR (2001) Fixation-based surgery: a new technique for distal radius osteotomy. Comput Aided Surg 6:160-169. https://doi.org/10. 3109/10929080109146002

5. Vroemen JC, Dobbe JGG, Strackee SD, Streekstra GJ (2013) Positioning evaluation of corrective osteotomy for the malunited radius: 3-D CT versus 2-D radiographs. Orthopedics 36:e193-e199. https://doi.org/10.3928/01477447-20130122-22

6. Stockmans F, Dezillie M, Vanhaecke J (2013) Accuracy of 3D virtual planning of corrective osteotomies of the distal radius. J Wrist Surg 2:306-314. https://doi.org/10.1055/s-0033-1359307

7. Fadero PE, Shah M (2014) Three dimensional (3D) modelling and surgical planning in trauma and orthopaedics. Surgeon 12:328-333. https://doi.org/10.1016/j.surge.2014.03.008
8. Dobbe JGG, Vroemen JC, Strackee SD, Streekstra GJ (2014) Patient-specific distal radius locking plate for fixation and accurate 3D positioning in corrective osteotomy. Strateg Trauma Limb Reconstr 9:179-183. https://doi.org/10.1007/s11751-014-0203-1

9. Dobbe JGG, Vroemen JC, Strackee SD, Streekstra GJ (2013) Patient-tailored plate for bone fixation and accurate 3D positioning in corrective osteotomy. Med Biol Eng Comput 51:19-27. https:// doi.org/10.1007/s11517-012-0959-8

10. Omori S, Murase T, Kataoka T, Kawanishi Y, Oura K, Miyake J, Tanaka H, Yoshikawa H (2014) Three-dimensional corrective osteotomy using a patient-specific osteotomy guide and bone plate based on a computer simulation system: accuracy analysis in a cadaver study. Int J Med Robot Comput Assist Surg 10:196-202. https://doi.org/10.1002/rcs.1530

11. Kataoka T, Oka K, Miyake J, Omori S, Tanaka H, Murase T (2013) 3-Dimensional prebent plate fixation in corrective osteotomy of malunited upper extremity fractures using a real-sized plastic bone model prepared by preoperative computer simulation. J Hand Surg Am 38:909-919. https://doi.org/10.1016/J.JHSA.2013.02.024

12. Vroemen JC, Dobbe JGG, Sierevelt IN, Strackee SD, Streekstra GJ (2013) Accuracy of distal radius positioning using an anatomical plate. Orthopedics 36:e457-e462. https://doi.org/10. 3928/01477447-20130327-22

13. Hoekstra H, Rosseels W, Sermon A, Nijs S (2016) Corrective limb osteotomy using patient specific 3D-printed guides: a technical note. Injury 47:2375-2380. https://doi.org/10.1016/j.injury.2016. 07.021

14. Kunz M, Ma B, Rudan JF, Ellis RE, Pichora DR (2013) Imageguided distal radius osteotomy using patient-specific instrument guides. J Hand Surg Am 38:1618-1624. https://doi.org/10.1016/j. jhsa.2013.05.018

15. Caiti G, Dobbe JGG, Strijkers GJ, Strackee SD, Streekstra GJ (2018) Positioning error of custom 3D-printed surgical guides for the radius: influence of fitting location and guide design. Int $\mathbf{J}$ Comput Assist Radiol Surg 13:507-518. https://doi.org/10.1007/ s11548-017-1682-6

16. Miyake J, Murase T, Moritomo H, Sugamoto K, Yoshikawa $\mathrm{H}$ (2011) Distal radius osteotomy with volar locking plates based on computer simulation. Clin Orthop Relat Res 469:1766-1773. https://doi.org/10.1007/s11999-010-1748-Z

17. Murase $T$ (2016) Surgical technique of corrective osteotomy for malunited distal radius fracture using the computersimulated patient matched instrument. J Hand Surg (Asian-Pacific) 21:133-139. https://doi.org/10.1142/S2424835516400051

18. Martelli N, Serrano C, Van Den Brink H, Pineau J, Prognon P, Borget I, El Batti S (2016) Advantages and disadvantages of 3-dimensional printing in surgery: a systematic review. Surg (United States) 159:1485-1500. https://doi.org/10.1016/j.surg. 2015.12.017

19. Tack P, Victor J, Gemmel P, Annemans L (2016) 3D-printing techniques in a medical setting: a systematic literature review. Biomed Eng Online 15:115. https://doi.org/10.1186/s12938-016-0236-4

20. Egger J, Gall M, Tax A, Ücal M, Zefferer U, Li X, von Campe G, Schäfer U, Schmalstieg D, Chen X (2017) Interactive reconstructions of cranial 3D implants under MeVisLab as an alternative to commercial planning software. PLoS ONE 12:e0172694. https:// doi.org/10.1371/journal.pone.0172694

21. Dobbe JGG, Strackee SD, Schreurs AW, Jonges R, Carelsen B, Vroemen JC, Grimbergen CA, Streekstra GJ (2011) Computerassisted planning and navigation for corrective distal radius osteotomy, based on pre- and intraoperative imaging. IEEE Trans Biomed Eng 58:182-190. https://doi.org/10.1109/TBME.2010. 2084576

22. Dobbe JGG, Strackee SD, Streekstra GJ (2018) Minimizing the translation error in the application of an oblique single-cut rotation 
osteotomy: where to cut? IEEE Trans Biomed Eng 65(4):821-827. https://doi.org/10.1109/TBME.2017.2721498

23. Mohammed MI, Fitzpatrick AP, Gibson I (2017) Customised design of a patient specific 3D printed whole mandible implant. KnE Eng 2:104. https://doi.org/10.18502/keg.v2i2.602

24. Modabber A, Ayoub N, Möhlhenrich SC, Goloborodko E, Sönmez TT, Ghassemi M, Loberg C, Lethaus B, Ghassemi A, Hölzle F (2014) The accuracy of computer-assisted primary mandibular reconstruction with vascularized bone flaps: iliac crest bone flap versus osteomyocutaneous fibula flap. Med Devices (Auckl) 7:211-217. https://doi.org/10.2147/MDER.S62698

25. Cormen TH, Leiserson CE, Riverst RL, Stein C (2001) Introduction to algorithms, 3rd edn. The MIT Press, Cambridge. https://doi.org/ 10.1017/CBO9781107415324.004

26. Taubin $G$ (1995) A signal processing approach to fair surface design. In: Proceedings 22nd annual conference on computer graphics and interactive techniques-SIGGRAPH'95. ACM Press, New York, NY, USA, pp 351-358

27. Schroeder W, Martin K, Lorensen B (2006) The VTK: an objectoriented approach to 3D graphics, 4th edn. Pearson Education Inc, London
28. Kukreja U, Stevenson WE, Ritter FE (2006) RUI: recording user input from interfaces under Windows and Mac OS X. Behav Res Methods 38:656-659. https://doi.org/10.3758/BF03193898

29. Banks J (2013) Adding value in additive manufacturing: researchers in the United Kingdom and Europe look to 3D printing for customization. IEEE Pulse 4:22-26. https://doi.org/10.1109/ MPUL.2013.2279617

30. Mulford JS, Babazadeh S, Mackay N (2016) Three-dimensional printing in orthopaedic surgery: review of current and future applications. ANZ J Surg. https://doi.org/10.1111/ans.13533

31. Wong KC, Kumta SM, Geel NV, Demol J (2015) One-step reconstruction with a 3D-printed, biomechanically evaluated custom implant after complex pelvic tumor resection. Comput Aided Surg 20:14-23. https://doi.org/10.3109/10929088.2015.1076039

32. Chen X, Xu L, Yang Y, Egger J (2016) A semi-automatic computeraided method for surgical template design. Sci Rep 6:20280. https:// doi.org/10.1038/srep20280

33. Chen X, Xu L, Li X, Egger J (2017) Computer-aided implant design for the restoration of cranial defects. Sci Rep 7:4199. https://doi. org/10.1038/s41598-017-04454-6 